\author{
Marquette University \\ e-Publications@Marquette
}

Biological Sciences Faculty Research and

Publications

Biological Sciences, Department of

$5-1999$

\title{
Commissural Interneurons in Rhythm Generation and Intersegmental Coupling in the Lamprey Spinal Cord
}

James T. Buchanan

Marquette University, james.buchanan@marquette.edu

Follow this and additional works at: https://epublications.marquette.edu/bio_fac

Part of the Biology Commons

\section{Recommended Citation}

Buchanan, James T., "Commissural Interneurons in Rhythm Generation and Intersegmental Coupling in the Lamprey Spinal Cord" (1999). Biological Sciences Faculty Research and Publications. 163.

https://epublications.marquette.edu/bio_fac/163 
Marquette University

e-Publications@Marquette

\title{
Biological Sciences Faculty Research and Publications/College of Arts and Sciences
}

This paper is NOT THE PUBLISHED VERSION; but the author's final, peer-reviewed manuscript. The published version may be accessed by following the link in the citation below.

Journal of Neurophysiology, Vol. 81, No. 5 (May 1999): 2037-2045. DOI. This article is (C) American Physiological Society and permission has been granted for this version to appear in $\underline{\mathrm{e}-}$

Publications@Marquette. American Physiological Society does not grant permission for this article to be further copied/distributed or hosted elsewhere without the express permission from American Physiological Society.

\section{Commissural Interneurons in Rhythm Generation and Intersegmental Coupling in the Lamprey Spinal Cord}

\author{
James T. Buchanan \\ Department of Biology, Marquette University, Milwaukee, Wisconsin
}

\begin{abstract}
Commissural interneurons in rhythm generation and intersegmental coupling in the lamprey spinal cord. To test the necessity of spinal commissural interneurons in the generation of the swim rhythm in lamprey, longitudinal midline cuts of the isolated spinal cord preparation were made. Fictive swimming was then induced by bath perfusion with an excitatory amino acid while recording ventral root activity. When the spinal cord preparation was cut completely along the midline into two lateral hemicords, the rhythmic activity of fictive swimming was lost, usually replaced with continuous ventral root spiking. The loss of the fictive swim rhythm was not due to nonspecific damage produced by the cut because rhythmic activity was present in split regions of spinal cord when the split region was still attached to intact cord. The quality of this persistent rhythmic activity, quantified
\end{abstract}


with an autocorrelation method, declined with the distance of the split spinal segment from the remaining intact spinal cord. The deterioration of the rhythm was characterized by a lengthening of burst durations and a shortening of the interburst silent phases. This pattern of deterioration suggests a loss of rhythmic inhibitory inputs. The same pattern of rhythm deterioration was seen in preparations with the rostral end of the spinal cord cut compared with those with the caudal end cut. The results of this study indicate that commissural interneurons are necessary for the generation of the swimming rhythm in the lamprey spinal cord, and the characteristic loss of the silent interburst phases of the swimming rhythm is consistent with a loss of inhibitory commissural interneurons. The results also suggest that both descending and ascending commissural interneurons are important in the generation of the swimming rhythm. The swim rhythm that persists in the split cord while still attached to an intact portion of spinal cord is thus imposed by interneurons projecting from the intact region of cord into the split region. These projections are functionally short because rhythmic activity was lost within approximately five spinal segments from the intact region of spinal cord.

\section{INTRODUCTION}

In lamprey, as in other vertebrates, the network of neurons generating the locomotor rhythm is located within the spinal cord. This is shown by the presence of rhythmic ventral root bursting in the isolated spinal cord when perfused with an excitatory amino acid such as glutamate (Cohen and Wallén 1980). The pattern of this rhythmic activity is similar to the pattern of myotomal electromyographic activity in the swimming lamprey and is therefore referred to as fictive swimming (Wallén and Williams 1984). Fictive swimming is characterized by an alternation of ventral root bursts on opposite sides of the spinal cord and a rostral-to-caudal propagation of the bursts. As few as two to three spinal segments taken from any rostral-caudal level can exhibit fictive swimming, so the locomotor network is conceived of as a chain of overlapping coupled oscillators (Cohen et al. 1992).

A proposed model (Fig. 1A) for the unit locomotor rhythm generator has at its core reciprocal inhibition between commissural interneurons located on opposite sides of the spinal cord (Buchanan 1986; Buchanan and Grillner 1987). This model is based on the patterns of synaptic interactions among interneurons revealed with paired intracellular microelectrode recordings (Buchanan 1982;Buchanan et al. 1989) and is similar to a model for the swim network in the newly hatched Xenopus spinal cord (Roberts 1990). One characterized class of commissural interneurons in lamprey is called CC interneurons because the main axon of these cells projects contralaterally and caudally, although they often also have a rostral-axonal bifurcation (Buchanan 1982). The model also contains excitatory interneurons (EINs) with ipsilateral axons (Buchanan et al. 1989) and inhibitory lateral interneurons with ipsilateral descending axons (Rovainen 1974). 

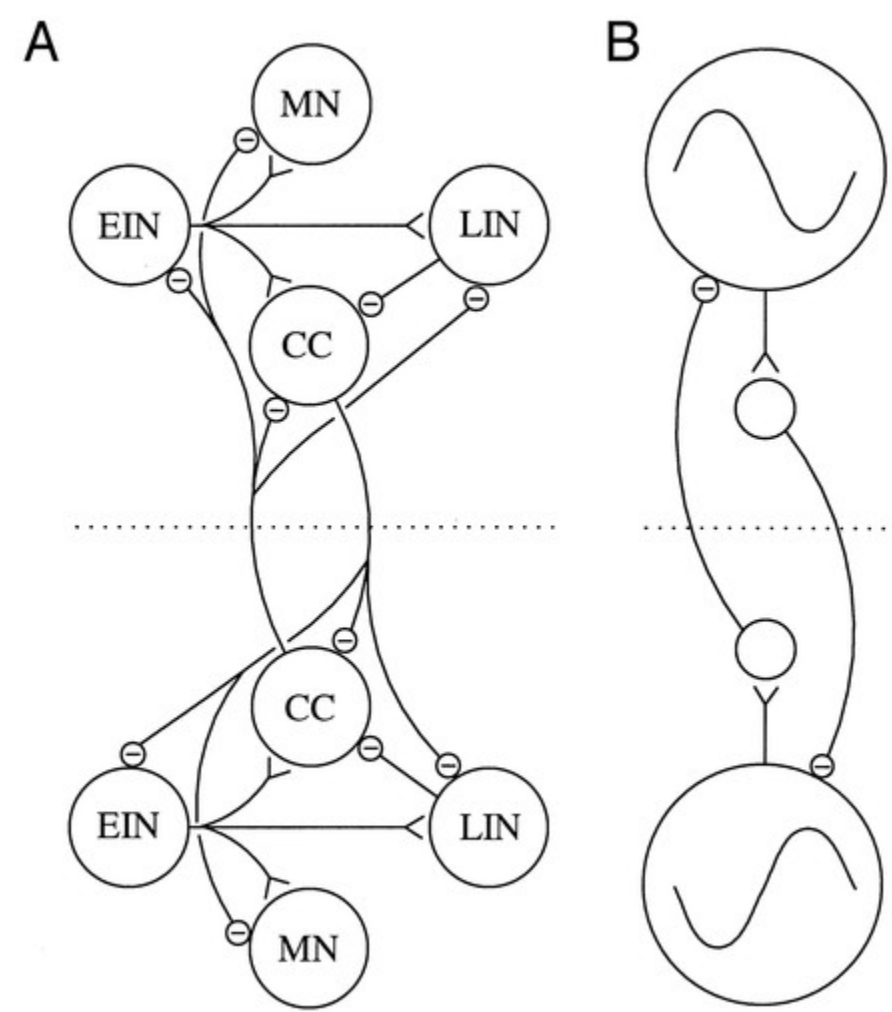

Fig. 1.Two alternative models for the role of commissural interneurons in fictive locomotion in the lamprey. $A$ : previously proposed model for the unit locomotor network (Buchanan and Grillner 1987). Inhibitory commissural interneurons (CC interneurons) are the core of the rhythm generator providing a reciprocal inhibitory network. Removal of the commissural interneurons abolishes rhythmic activity. Circles represent populations of neurons, and the dotted line is the midline. Forked terminals are excitatory synapses; circles with minus signs are inhibitory synapses. CC: interneurons with caudal and contralateral axons; MN: motoneurons; EIN: excitatory interneurons; LIN: lateral interneurons. B: alternative model in which each side of the spinal cord has an autonomous oscillator of unknown structure. Inhibitory commissural interneurons serve to antiphasically couple the oscillators. Removal of commissural interneurons does not abolish rhythmic activity but uncouples the rhythmic activity of the 2 sides of the cord.

For the proposed network model (Fig. 1A), rhythmogenesis originates mainly from the reciprocal inhibition between commissural interneurons on opposite sides of the cord. Activity in commissural interneurons on one side inhibits the activity of cells on the opposite side, and when these inhibited commissural interneurons escape or are released from inhibition (Wang and Rinzel 1992) they become active and inhibit cells of the previously active side. A key to the functioning of reciprocal inhibition as a rhythm generator is the mechanism for this escape or release. Within the network model of Fig.1A, the lateral interneurons provide a feedforward inhibition that can terminate ipsilateral CC interneuron activity, but other burst terminating mechanisms have been proposed such as accumulation of calcium-activated potassium conductance (El Manira et al. 1994). However, for the network model of Fig.1A, rhythmicity is lost when the outputs of commissural interneurons are removed (Buchanan 1992).

An alternative to the proposed network model of Fig. $1 A$ is one in which each side of the spinal cord contains independent rhythm generators, coupled via commissural interneurons (Fig. 1B). In this model, the inhibitory commissural interneurons produce antiphasic coupling of the two sides but are not essential to rhythm generation. Rhythm generation in this model would be the result of cellular and network properties that are as yet not known in lamprey. The model of Fig. $1 B$ predicts that removal of the outputs of commissural 
interneurons would not abolish rhythmic activity but would only uncouple the rhythm generators on each side of the spinal cord.

The necessity of commissural interneurons for rhythm generation has not been clearly established in the lamprey. One approach to this question has been to apply strychnine to the spinal cord because the output inhibitory postsynaptic potentials of commissural interneurons are glycinergic and are blocked by strychnine (Buchanan 1982; McPherson et al. 1994). Application of strychnine (5 $\mu \mathrm{M})$ can speed fictive swimming and ultimately eliminate rhythmicity (Grillner and Wallén 1980;McPherson et al. 1994), or it can lead to synchronous rhythmic activity on the two sides of the spinal cord (Alford and Williams 1989; Cohen and Harris-Warrick 1984; Hagevik and McClellan 1994). Lower concentrations of strychnine (0.1-0.2 $\mu \mathrm{M})$ can produce a slow modulation of the swimming rhythm that maintains an alternating pattern (McPherson et al. 1994). Thus the results of strychnine experiments are mixed with regard to the issue of the necessity of commissural interneurons. One problem with strychnine application is that it blocks almost all synaptic inhibition in the spinal cord, not just commissural inhibition (Homma and Rovainen 1978), and may also block GABA receptors (Baev et al. 1992). Strychnine in the 1- to $10-\mu \mathrm{M}$ range is not selective for ligand-gated receptors but can also reduce voltage-gated sodium and potassium currents (Shapiro et al. 1974) and voltage-gated calcium currents (Oyama et al. 1988). Strychnine application may thus result in rhythmic activity that is not directly relevant to activity in the locomotor network. The most direct test of the necessity for commissural interneurons is to make midline cuts that completely isolate the spinal cord into lateral halves (hemicords). It has been reported that lateral hemicords of lamprey can show rhythmic activity in the presence of strychnine (Grillner et al. 1986), but again it is not clear to what extent this strychnine-dependent rhythmic activity relies on the locomotor network.

In this study, midline cuts were made on the lamprey spinal cord to assess the necessity of commissural interneurons in the production of fictive swimming. The cuts were first done partially, beginning at one end of the spinal cord piece and extending to approximately one-half the length of the piece. This partial cut was done to determine whether the midline cut produced nonspecific damage that could block rhythmic activity. These partial midline cuts yielded preparations in which rhythmic activity was present in the split spinal cord located near the intact cord but was absent in more distant split segments. After complete isolation of the two lateral halves of the spinal cord, the rhythmic activity of fictive swimming was lost. These experiments not only demonstrate that nonspecific damage is insufficient to explain the loss of rhythmic activity but also provide insight into the lengths of the coupling signals among the locomotor rhythm generators.

The current experiments support the proposal that the commissural interneurons are necessary for the generation of the swim rhythm in lamprey and suggest that the functional lengths of the coupling connections are relatively short, on the order of approximately five segments.

\section{METHODS}

The experiments were done on seven adult silver lampreys (Ichthyomyzon unicuspis), $18-30.5 \mathrm{~cm}$ in length. Each animal was anesthetized by immersion in a $0.01 \%$ solution of tricaine ( 3 -aminobenzoic acid ethyl ester) (Sigma) until reflexes were lost. The animal was then decapitated, the brain was destroyed mechanically, and a spinal cord-notochord preparation was dissected in cooled Ringer solution as previously described (Rovainen 1974). The Ringer solution consisted of the following (in $\mathrm{mM}$ ): $91 \mathrm{NaCl}, 2.1 \mathrm{KCl}, 2.6 \mathrm{CaCl}_{2}, 1.8 \mathrm{MgCl}_{2}$, 4 glucose, and 20 $\mathrm{NaHCO}_{3}$. The solution was continuously bubbled with $98 \% \mathrm{O}_{2}-2 \% \mathrm{CO}_{2}(\mathrm{pH} 7.4)$, and the preparation was perfused at a rate of $2-4 \mathrm{ml} / \mathrm{min}\left(8-9^{\circ} \mathrm{C}\right)$.

For these experiments, the spinal cord-notochord preparations typically consisted of 22 segments (range 1450). However, no systematic differences were observed between preparation length and the parameters measured in this paper. The spinal cord pieces came mainly from the midbody of the lamprey, with some 
extension into the fin region in three pieces, and some extension into the gill region in two pieces. One preparation came entirely from the fin region. Once a preparation was pinned to the Sylgard-lined floor of the experimental chamber and perfused with normal Ringer solution, a longitudinal cut was made along the midline with either the tip of a 30-gauge needle or with fine spring scissors. The cuts extended either from the caudal end $(n=5)$ or from the rostral end $(n=5)$ to $\sim 40 \%$ of the length of the spinal cord piece (range $33-50 \%)$. For graphic convenience, the ventral root in the segment where the longitudinal cut began was numbered 0 , and segments in the intact region were given sequential negative numbers, and segments in the cut region were given sequential positive numbers.

After the longitudinal midline cut was made, fictive swimming was induced by bath perfusion with $N$-methyl-d, Iaspartate (NMA) (0.2 to $0.3 \mathrm{mM}$ ) or with $N$-methyl-d-aspartate (NMDA) (0.15-0.2 mM). The resulting swim cycle periods ranged from 0.41 to $1.22 \mathrm{~s}$, mean $=0.67 \pm 0.22(\mathrm{SD})$. Three ventral roots were monitored by placing the tips of glass suction electrodes onto ventral roots near their exit points from the spinal cord (Fig.2, $A$ and $B$ ). Two of the electrodes were stationary and were placed on opposite sides of the spinal cord in the intact region, and a third electrode was moved from root to root along the spinal cord. Ventral roots on one side of the cord were sampled with the roving electrode with recordings of 1-min duration each. Then, while recording from a ventral root in the split cord region located near the intact cord, a transverse cut was made at the beginning of the longitudinal midline cut on the recorded side to isolate that lateral hemicord (see Fig. 5 for a schematic drawing of these cuts). Activity of ventral roots in the isolated hemicord was then sampled. A similar procedure was then carried out on the opposite side of the cord. The ventral root recordings were band-pass filtered $(50-2,000 \mathrm{~Hz})$ and stored on an eight-channel DAT recorder (Biologic) for later off-line analysis. For the off-line analysis, the ventral root signals were low-pass filtered with a cutoff frequency of $1,000 \mathrm{~Hz}$ and digitized at 2,000 $\mathrm{Hz}$ with a Cambridge Electronic Design 1401 computer interface, Spike2 software (CED), and a 486 computer.

A
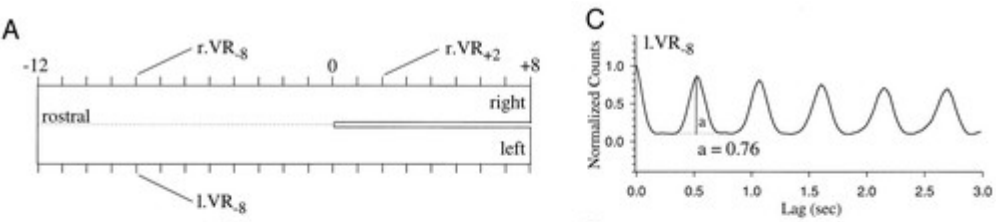

B

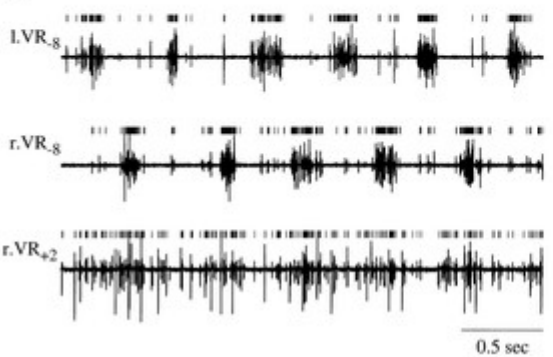

D
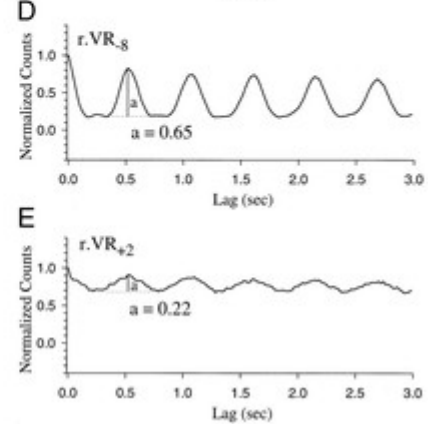

Fig. 2. Method for measuring the effect of longitudinal spinal hemisections on the quality of the fictive swimming rhythm. A: diagram of a 20-segment length of isolated spinal cord split along the midline for 8 segments at the caudal end. The ventral root (VR) nearest to the beginning of the cut was numbered 0 , and ventral roots within the intact region of spinal cord were given sequential negative values, whereas those within the split region were given positive values. Ventral roots used for the recordings illustrated in Bare indicated. $B$ : fictive swimming was induced by application of $N$-methyl-d-aspartate $(0.15 \mathrm{mM})$ to the bath, and the rhythmic bursting of ventral roots was recorded with glass suction electrodes. Shown are recordings on the left and right sides of the spinal cord in the intact region $\left(\mathrm{I} \mathrm{VR}_{-8}\right.$ and $\mathrm{r}$. $\left.\mathrm{VR}_{-8}\right)$ and a ventral root within the split region of cord on the right side $\left(r . \mathrm{VR}_{+2}\right)$. Action potentials were converted to event times by thresholding on a voltage just above the noise level of the recording. These event times are illustrated above the raw ventral root recordings. Time calibration bar applies to all 3 traces. C-E: autocorrelations were calculated on the event times for 1 min of ventral root activity. For each event, the number of events occurring in consecutive 20 -ms bins was counted 
over a 3-s window (150 bins). This procedure was repeated for all events, and the resulting counts were summed for each bin. The counts were normalized to the maximum bin count. This procedure produced a periodic function with the same cycle period as the rhythmic activity in the roots. To quantify the quality of the rhythm, the amplitude (a) of the 2 nd peak of the autocorrelation was measured from its base (defined as the mean of the troughs on either side) to its peak. This measure was defined as the quality of rhythmic activity (QRA). QRA was high in $\mathrm{I}_{\text {. } V R_{-8}}(C)$ and $r$. $\mathrm{VR}_{-8}(D), 0.76$ and 0.65 , respectively, but low in $r$. $\mathrm{VR}_{+2}(E), 0.22$. These values correspond to the general impression obtained from the raw recordings in $B$.

To quantify the quality of rhythmic activity in the ventral root recordings, an autocorrelation was performed on the event times of the ventral root action potentials. For this, the action potentials were converted to event times by setting a voltage threshold so that each time the voltage rose above the threshold level an event time was recorded. This threshold value was set just above the intrinsic noise level of the ventral root recording (Fig. 2B). An autocorrelation was performed on these event times with software provided by Spike2. For each event, the numbers of events occurring in consecutive 20-ms bins were counted over a 3-s time period (150 bins); this counting procedure was repeated for all events in the $1 \mathrm{~min}$ of recorded activity, and the resulting counts were summed for each bin. The counts were normalized to the maximum bin count (i.e., the first bin).

The resulting autocorrelation was a decaying oscillation (Fig. 2,C-E). The period of the autocorrelation oscillation was the same as the mean cycle period of fictive swimming. The oscillations became smaller in trough-to-peak amplitude because of lower peaks and higher troughs. The fall in the peaks is due to irregularity of the cycle periods so that sequential bursts do not exactly align; the rise in the troughs is due to the presence of spikes occurring between the ventral root bursts. The degree of attenuation of the trough-to-peak amplitudes of the oscillations in the autocorrelation gives an overall measure of rhythm quality, i.e., the regularity of the swim cycle period and the degree of confinement of spiking to the bursts. Therefore, to provide a measure of the quality of rhythmic ventral root bursting, the trough-to-peak amplitude of the second peak of the autocorrelation was used (Fig. 2C). The base of the peak was defined as the mean of the minimum values of the first and second troughs, and the amplitude from this level to the peak was measured (Fig. 2, C-E). The second peak amplitude of the autocorrelation is referred to in this paper as the quality of rhythmic activity or (QRA) and could potentially range from 0 to 1.0. Actual measured values ranged from 0.01 to 0.94 .

\section{RESULTS}

\section{Partial midline cuts}

Spinal cord preparations were first cut along the midline from one end to $\sim 40 \%$ of the total length of the piece. When NMDA (or NMA) was then applied to the bath, rhythmic ventral root bursting was induced. In the intact region of spinal cord, this bursting was regular with relatively few spikes occurring between bursts, as is typical of fictive swimming in nonlesioned spinal cord (Fig. 2B). In contrast, ventral root bursting was less obvious within the region of spinal cord in which the two sides of the cord were separated by the longitudinal midline cut (i.e., the split region). Although some rhythmicity was apparent, the distinction between bursts and interburst periods was less clear. To quantify the quality of rhythmic activity, autocorrelations of the action potential event times were performed, and the normalized trough-to-peak amplitudes of the second peak of the autocorrelation were measured. This amplitude is referred to as the QRA. In the particular experiment of Fig. 2, the ventral roots in the intact region of cord had QRA values of 0.76 and 0.65 for the left and right sides of the spinal cord

(Fig. 2, $C$ and $D$ ). The QRA of a ventral root within the split region of cord had a smaller amplitude of 0.22 (Fig. 2E).

Systematic recording of ventral roots in a spinal cord piece revealed that the quality of rhythmic bursting in the split region decreased with the distance between the ventral root and the beginning segment of the longitudinal 
midline cut. For example, in Fig.3 the ventral root located in the intact cord just rostral to the beginning of a caudally directed longitudinal midline cut $\left(\mathrm{VR}_{-1}\right)$ had distinct bursts and a QRA of 0.7 (Fig. $3 A$ ). In the split region, the bursts became progressively less distinct in ventral roots located further from the intact region of cord (compare $\mathrm{VR}_{+1}, \mathrm{VR}_{+2}, \mathrm{VR}_{+3}$, and $\mathrm{VR}_{+7}$ in Fig. $3, A$ and $C$ ). In this particular experiment, the rhythm in $\mathrm{VR}_{+3}$ was barely apparent ( $Q R A=0.07)$, whereas at $\mathrm{VR}_{+7}$ no rhythmic activity was detectable in the autocorrelation. The $\mathrm{QRA}$ of $\mathrm{VR}_{+7}$ was 0.04 , which reflects the noise level of the autocorrelation.
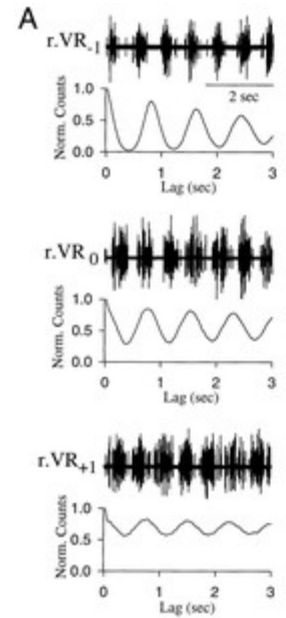
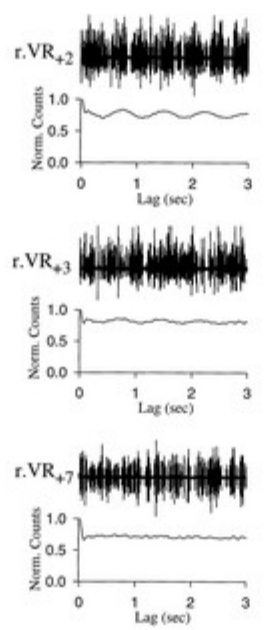

B
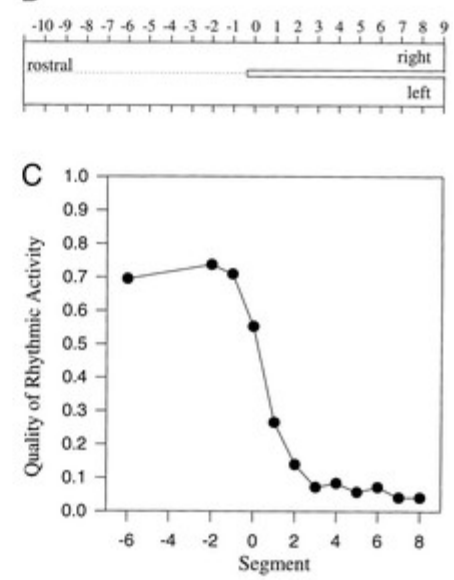

Fig. 3. Relationship of rhythmic quality with distance from the intact region of spinal cord. $A$ : samples of raw ventral root recordings and their autocorrelations from the right side of the spinal cord at various rostral-caudal levels. The time calibration bar applies to all ventral root traces. $B$ : spinal cord piece for this experiment was 20 segments in length and was split for 9 segments at the caudal end. As can be appreciated both in the raw recordings and in the autocorrelation functions, there was a deterioration in the quality of the rhythm in the split segments, and this deterioration was progressive with distance from the intact region of cord. Deterioration was characterized by a lengthening of burst durations and a shortening of silent periods. $C$ : plot of the amplitude of the 2 nd peak of the autocorrelation function (i.e., QRA) vs. the segment location of the ventral root recording. When no rhythm could be detected, as in $r$. $V R_{+7}$, the amplitude measurement reflects the noise level of the autocorrelation.

As summarized in Fig. 4, the longitudinal midline cuts reduced the quality of the swim rhythm in all preparations, and the effect was progressive with the distance of the split segment from the intact region of spinal cord. The data are divided into those preparations with the rostral end cut (Fig.4A) and those with the caudal end cut (Fig. 4B). Although midline cuts produced reductions in rhythm quality in the intact region of both types of preparations, those with the rostral end cut generally had poorer rhythms in the intact region than did those with the caudal end cut (Fig. 4C). In spite of this difference in the quality of rhythmic activity in the intact region, the fall in rhythm quality in the split region with distance showed a similar relationship in the two preparations (Fig. 4D). 

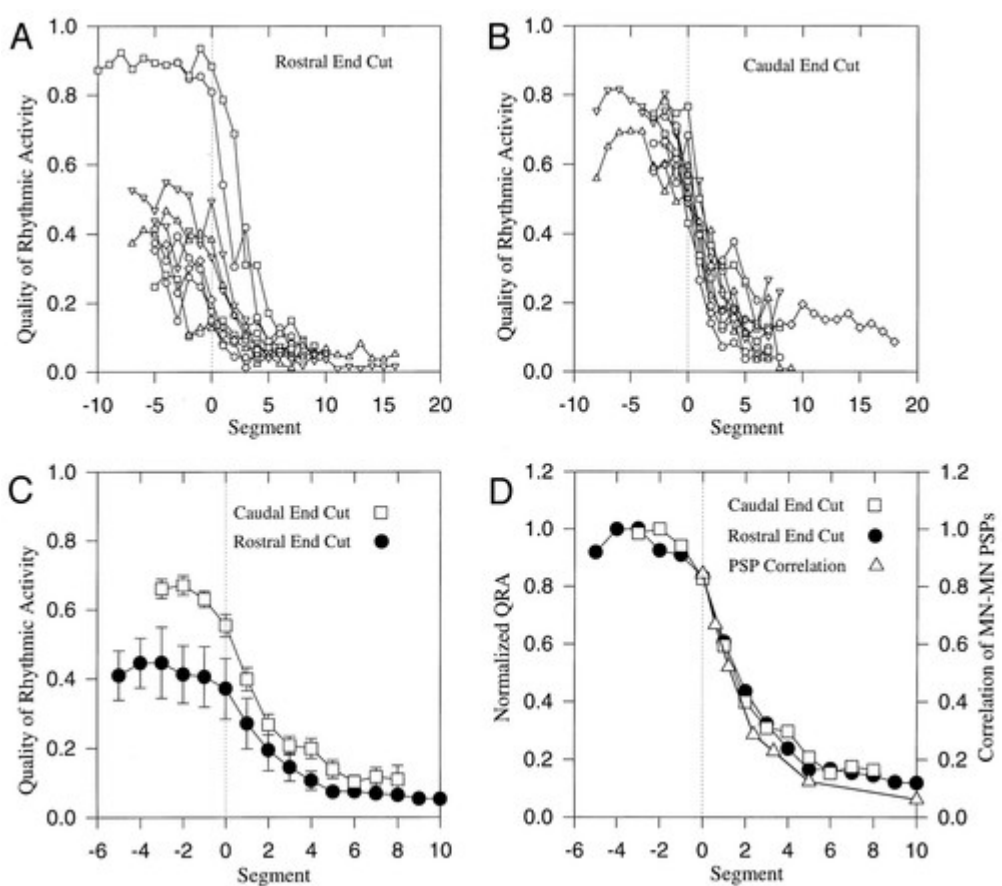

Fig. 4.Summary of the relationship of QRA vs. ventral root location. As in previous figures, segment 0 is the ventral root closest to the beginning of the cut. $A$ : 10 experiments in 5 preparations in which the rostral end of the cord was cut. $B$ : 10 experiments in 5 preparations in which the caudal end was cut. In both cases, the quality of the rhythmic activity dropped rapidly to low levels within $\sim 5$ segments. In general, the quality of the rhythm in the intact region of cord was higher in the cases in which the caudal end was cut compared with when the rostral end was cut. One preparation with the rostral end cut was an exception to this finding. $C$ : means $\pm S E$ of the 10 experiments with the rostral end cut $(\bullet)$ and the 10 experiments with the caudal end cut $(\boldsymbol{\square})$ are shown.D: 2 plots of $C$ have each been normalized to each curve's maximum value. The fall in the QRA with distance is similar whether the cut was made at the caudal or at the rostral end. A third plot $(\Delta)$ has been added from previous results showing the relationship of postsynaptic potential cross-correlations vs. the number of segments between motoneurons (Buchanan et al. 1995).

\section{Complete midline cuts}

When the split region of spinal cord was completely separated from the intact region by making a transverse hemisection at the beginning of the longitudinal midline cut, the rhythmic activity of fictive swimming was always eliminated in the ventral roots of the isolated lateral hemicord. For example, in Fig.5A a ventral root recording in the split region of cord (right $\mathrm{VR}_{+1}$ ) displayed rhythmic activity while still attached to the intact spinal cord. The rhythmicity was apparent both in the raw ventral root activity (Fig.5A2) and in the autocorrelation (Fig. 5A3). When a transverse cut was made to completely isolate the right split region from the intact cord (Fig. 5B1), rhythmic activity was lost in the right hemicord. Again the loss of rhythmicity was apparent both in the raw ventral root activity (Fig. 5B2) and in the autocorrelation (Fig. 5B3). The same result was observed when the other one-half of the split region of cord was severed (compare Fig. 5, $C$ and $D$ ). 

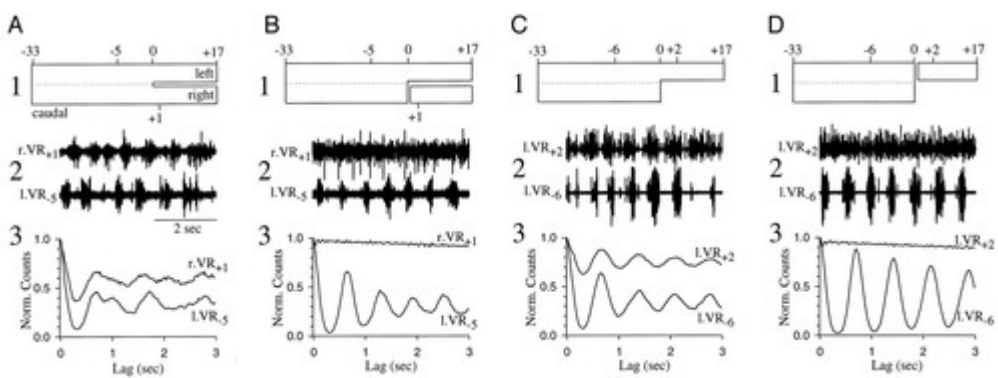

Fig. 5.Effect of complete hemicord isolation on rhythmic activity.A1: spinal cord piece of this experiment consisted of 50 segments with the midline cut at the rostral end and extending 17 segments. A2: sample of raw ventral root recordings from $\mathrm{r}$. $\mathrm{VR}_{+1}$ and $\mathrm{I}_{\mathrm{V}} \mathrm{VR}_{-5}$. A3: autocorrelation functions of the 2 ventral root recordings showing low-quality rhythms in both roots. $B 1$ : transverse cut was made on the right side, completely isolating the right rostral 17 hemisegments. $B 2$ : ventral root within the completely isolated hemicord $\left(r . R_{+1}\right)$ lost rhythmic activity, whereas ventral root within in the intact region $\left(\mathrm{I} \mathrm{VR}_{-5}\right)$ showed an improved rhythm. B3: autocorrelations corroborate the impression from the raw records. C1-C3: ventral root recordings were changed to the $\mathrm{I}_{\mathrm{V}} \mathrm{VR}_{-6}$ in the intact regions and $\mathrm{I}_{\mathrm{V}} \mathrm{VR}_{+2}$ in the split region, and raw recordings and autocorrelation functions from these ventral roots are shown. D1: transverse cut was made to isolate the left rostral 17 hemisegments. $D 2$ and $D 3$ : again rhythmic activity was lost in the isolated hemicord, whereas there was an improvement in the quality of the rhythm in the intact region.

A second effect of removing the split region of cord was that the quality of rhythmic activity in the intact region of cord tended to improve. For example, in Fig. 5A the QRA in the intact region (left VR-5) showed an improvement from 0.24 to 0.59 after the right one-half of the split region was removed (Fig. $5, A 3$ vs. B3). A further improvement was observed when the remaining left one-half was removed from 0.51 to 0.85

(Fig. 5,C3 vs. D3).

In 6 of 19 experiments, some rhythmic activity was apparent in the isolated hemicord but was not considered to be a remnant of the fictive swim rhythm because it had a much higher frequency than the fictive swim rhythm. For example, in Fig. 6 a ventral root located on the right side in the split region $\left(r\right.$. $\left.V R_{+3}\right)$ showed the fictive swim rhythm while still attached to the intact cord (Fig. 6A), but after a transverse cut isolated the right hemicord (Fig. 6B) the fictive swim rhythm of 0.70 -s cycle period was lost in $r$. $\mathrm{VR}_{+3}$. However, rhythmic activity with a cycle period of $0.22 \mathrm{~s}$ was at times present in both the raw ventral root activity (Fig. 6B2) and in the autocorrelation (Fig. 6B3). This faster rhythm appeared to have been present weakly before the isolation of the hemicord as an intraburst rhythm (* in Fig. 6A3).
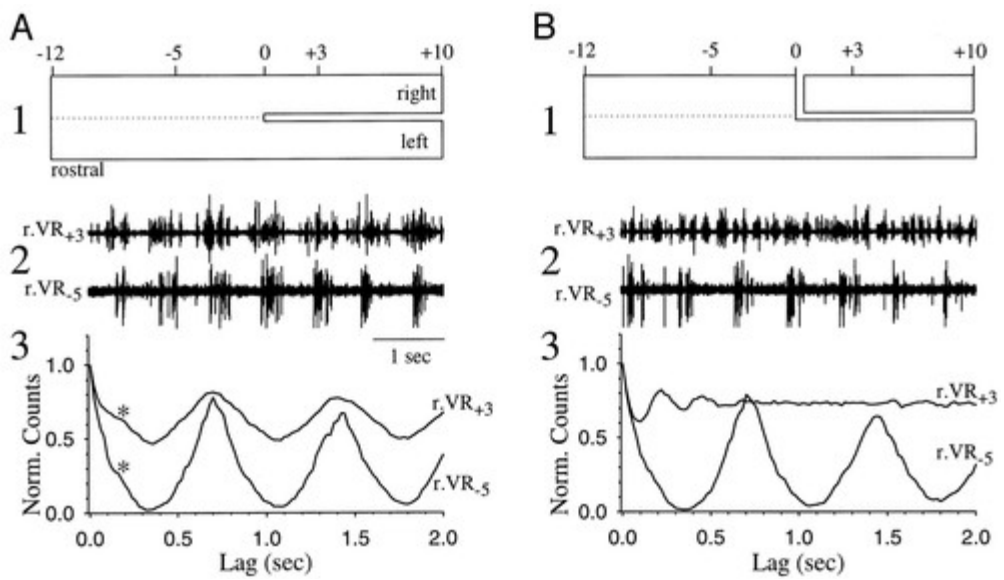

Fig. 6. Example of hemicord isolation resulting in a higher-frequency rhythm.A1: 22-segment length of cord was used that was split at the caudal end for 10 segments. $A 2$ : raw ventral root activity showing bursting with a 
period of $0.7 \mathrm{~s}$ in the intact and split region. $A 3$ : both ventral roots show periodic autocorrelations with the same period as the swim rhythm. A weak shorter-interval component (at $\sim 0.17 \mathrm{~s}$ ) caused by an intraburst rhythm is indicated by *. B1: transverse cut on the right side was made to isolate the right caudal hemicord.B2: rhythmic activity with a higher frequency than fictive swimming was observed in the completely isolated hemicord $(r$. $\left.\mathrm{VR}_{+3}\right) . B 3$ : autocorrelation showed that the intact region maintained the swim rate at the original cycle period of $0.7 \mathrm{~s}$, whereas the ventral root in the isolated hemicord had a period of $0.22 \mathrm{~s}$. This experiment exhibited the strongest rhythm observed in the 6 preparations showing the fast rhythm in the isolated hemicord.

A summary of the effects of isolation of the lateral hemicords is shown in Fig. 7. The fictive swimming rhythm was lost in all 19 cases, and no rhythmic activity was detectable in 13 of 19 cases. In these 13, the QRA at the presumed time of the fictive swim cycle period was $0.03 \pm 0.02( \pm S D)$, which represents the noise level of the autocorrelation. In 6 of 19 cases (circles with dots in Fig. 7A), there was a higher-frequency rhythm present after the transverse cut. This rhythm was weak and had a mean QRA of $0.11 \pm 0.05(n=6)$. A comparison of the cycle periods before and after the transverse cut is shown in Fig.7B for these six preparations. The cycle period decreased from a mean of $0.74 \pm 0.31 \mathrm{~s}$ before the cut to $0.20 \pm 0.039 \mathrm{~s}$ in the isolated lateral hemicord.
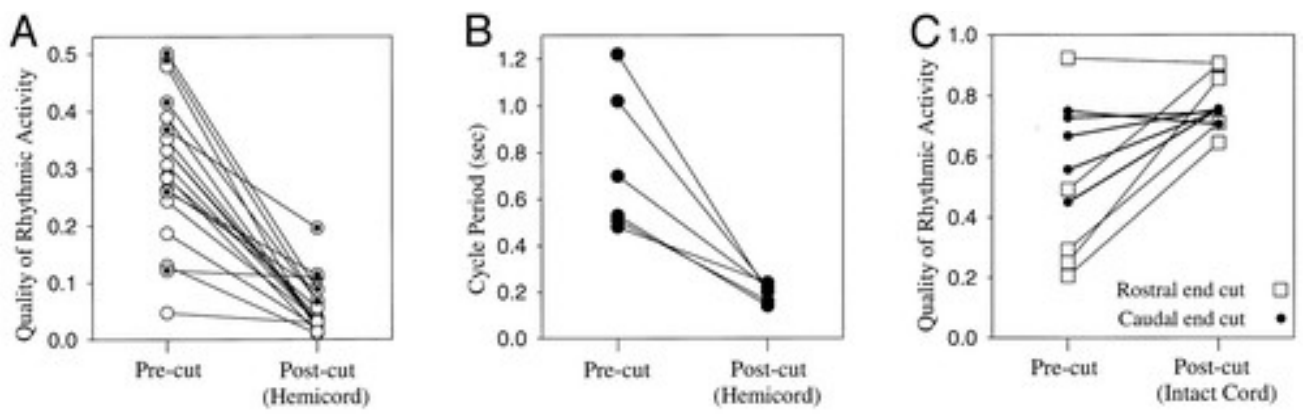

Fig. 7.Summary of the effects of complete hemicord isolation.A: changes in the quality of rhythmic activity in split regions of cord before complete isolation (Precut) and after making a transverse cut to isolate the hemicord (Postcut). Open circles without dots: experiments in which all apparent rhythmic activity was lost; open circles with dots: experiments with some rhythmic activity remaining. Ventral roots recorded were usually located at $\mathrm{VR}_{-2}$ or $\mathrm{VR}_{-3}$. $B$ : cycle periods before and after the transverse cut in the 6 preparations that showed some rhythmic activity after complete isolation of the hemicord (i.e., the preparations indicated by circles with dots in $A$ ). Although the swim cycle periods varied from 0.5 to $1.2 \mathrm{~s}$, the fast rhythm had a consistent cycle period of $\sim 0.2 \mathrm{~s}$. $C$ : effect of the removal of both of the split halves on QRA in the intact part of the cord. Generally, the spinal cords with the rostral end cut (open squares) had lower-quality rhythms in the intact region than the preparations with the caudal end cut (solid circles). After removal of the split regions, QRAs improved, suggesting that interneurons from the split region were disrupting the rhythm generators in the intact region. Because removal of the rostral end had greater improvement than removal of the caudal end, it would appear that descending interneurons have stronger effects or effects acting over longer distances on the rhythm generators than do ascending interneurons.

Figure $7 C$ shows the increase in rhythm quality in the intact cord after removal of the split regions for all preparations. Overall, the QRA increased from $0.53 \pm 0.24$ to $0.77 \pm 0.087$ ( $P=0.008$, Student'st-test) after both split regions were removed. Because the preparations with the rostral end split began with poorer rhythms, they tended to show greater improvements after removal of the rostral hemicords. The preparations with the rostral ends cut improved from 0.43 to 0.80 , whereas the preparations with the caudal ends cut improved from 0.63 to 0.74 . 


\section{DISCUSSION}

\section{Commissural interneurons and rhythm generation}

These experiments demonstrated that neurons with axons crossing the midline are necessary for the generation of the swim rhythm in the lamprey spinal cord. This was shown by the loss of the swim rhythm when one side of the spinal cord was completely isolated by a longitudinal midline cut (Fig. 5). The loss of the swim rhythm in the hemicord was not due to nonspecific cell damage when the midline cut was made. This was shown by the persistence of the swim rhythm in split segments as long as the split segments were still attached to intact spinal cord. Thus, with regard to the two alternatives posed in Fig. 1, the present experiments support Fig. 1A; commissural interneurons are necessary for rhythm generation rather than simply providing antiphasic coupling of autonomous rhythm generators located on each side of the cord (Fig. 1B).

Typically, the isolated hemicords exhibited continuous ventral root spiking in the presence of NMDA, suggesting that the midline cuts disrupted rhythmic activity by eliminating rhythmic inhibition. Further support for a loss of rhythmic inhibitory inputs comes from the characteristic pattern of deterioration of the swim rhythm in spinal cords with partial cuts. With greater distance from the intact region of cord, the ventral root burst durations increased whereas silent period durations decreased, suggesting that with distance there is a progressive loss of the inhibitory signals that sculpt out the silent inhibitory phases (Figs. 2 and 3). Inhibitory commissural interneurons, i.e., CC interneurons, have been characterized in the lamprey spinal cord (Buchanan 1982) and are likely to be a main source for the rhythmic inhibition occurring during fictive swimming (Fig. $8 \mathrm{~A}$ ). Because cells with ipsilateral projections were still intact, ipsilateral inhibitory interneurons (Buchanan and Grillner 1988) appear not to be the main source of rhythmic inhibition during fictive swimming.
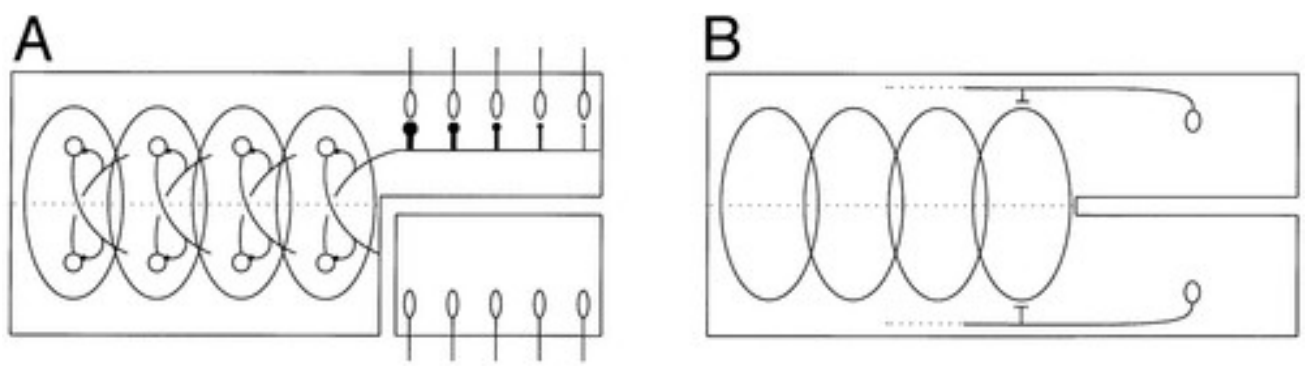

Fig. 8.Schematic summary of the conclusions. A: lamprey spinal cord contains an overlapping chain of rhythm generators, each producing the basic alternating rhythm. The results suggest that commissural interneurons are essential for the generation of the swim rhythm because the swim rhythm is always lost when the hemicord is completely isolated. The results also suggest that the key commissural interneurons are inhibitory (represented as $\bullet$ ) because the deterioration of the rhythm is characterized by longer-duration bursts and shorter silent periods as if the signal responsible for burst termination has been lost. Because the ventral root burst quality deteriorates with distance, it is proposed that the inhibitory signals coming from the intact central pattern generators have stronger influences locally and that their effects become weaker with distance (depicted by the decreasing size of lines and $\bullet$ ). Whether this decline in strength is due to characteristic lengths of the projecting axons or to a fall in synaptic strengths in individual axons with distance from the cell body is not known.

Although not shown, the results of the study indicate that the commissural interneurons project equally in the ascending and descending directions. $B$ : because the quality of the rhythmic activity in the intact region improves when the split regions of cord are removed, it seems likely that interneurons project into the intact region via ipsilateral axons. Because these cells are carrying abnormal signals caused by the deterioration of rhythm generation in the intact region, they have a disruptive effect. It is not known whether these signals are excitatory or inhibitory. 
Another important outcome of this study is that both ascending and descending commissural interneurons are important for the production of rhythmic activity. This was shown by the persistence of rhythmic activity in split regions regardless of whether the rostral or the caudal end was split (Fig. 4). This rhythmic activity would be due to ascending or descending commissural projections, respectively. Also, the quality of rhythmic activity degrades equally with distance in the two cases, suggesting that the ascending and descending commissural influences are of comparable strength and distance.

In a few cases, the isolated hemicord displayed an irregular rhythm that had a much higher frequency than that of fictive swimming (Fig.6). This fast rhythm was similar in frequency to intraburst rhythms sometimes observed during fictive swimming (Fig. 6). These intraburst rhythms may be due to intrinsic tendencies toward oscillatory membrane potential activity in individual motoneurons, especially in the presence of NMDA (Moore et al. 1993; Murphey et al. 1995). For example, in the presence of TTX, NMDA induces oscillatory membrane potentials (Wallén and Grillner 1987). These NMDA-induced, TTX-resistant oscillations have a cycle period of $\sim 1$ $\mathrm{s}$, but often a faster oscillation $(\sim 10 \mathrm{~Hz})$ is present on the plateau phase (Tabak et al. 1995). Even if intrinsic cellular mechanisms are responsible, there must also be some mechanism for synchronization, and this may be due to electrical coupling among motoneurons (Buchanan et al. 1998) or to highly interconnected premotor excitatory inputs to the motoneurons (Buchanan et al. 1989). Similarly, rhythmic bursting in the presence of strychnine, in which most inhibition has been blocked, may also be due to synchronization of the depolarization of populations of neurons followed by intrinsic repolarizing response caused by perhaps strong activation of potassium currents.

Further support for the conclusion that commissural interneurons are necessary for rhythm generation comes from photoablation studies on the lamprey spinal cord (Buchanan and McPherson 1995). In these experiments, commissural interneurons on one side of the spinal cord were retrogradely labeled with eosin-dextran amines. Intracellular recordings of labeled neurons demonstrated that illumination with an argon laser depolarized the cells and blocked the action potential within several minutes. During fictive swimming, laser illumination of the side of the cord with the retrogradely labeled commissural interneurons could abolish rhythmic activity. The deterioration in the rhythm was progressive and showed a characteristic pattern; ventral root bursts on the nonilluminated side increased in duration whereas the interburst silent intervals shortened; ventral root bursts on the illuminated side decreased in duration whereas the interburst silent periods lengthened. This pattern is consistent with the loss of inhibitory commissural interneurons on the illuminated side, resulting in less inhibitory sculpting of motoneuron firing on the nonilluminated side and greater inhibition of motoneurons on the illuminated side from the disinhibited commissural interneurons on the nonilluminated side.

\section{Functional length of multisegmental signals}

The fictive swim rhythm present in the split region of the cord when still attached to intact cord is imposed by rhythmic inputs coming from neurons within the intact region of cord because it is abolished when these inputs are removed (Fig. 5). As argued previously, these inputs are most likely to be from inhibitory commissural interneurons. The fall in rhythm quality in the split region with distance from the intact cord suggests that the cells in the intact region that project into the split cord have a limited length of influence (Fig. $8 A$ ). The relationship of rhythm quality versus distance may thus give an overall measure of the functional length of these multisegmental projections. Within approximately five segments, rhythmic activity was lost in the split region (Fig. 4), suggesting that after five segments the overall strengths of the projections (number of axons and their individual synaptic strengths) were too weak to modulate motoneuron firing. The functional lengths of the ascending and descending projections appear to be similar (Fig.4D).

The distance relationship found in this work matches well the one observed in a separate study (Buchanan et al. 1995 ) in which the correlation of synaptic potentials between motoneurons separated by various distances 
showed a similar fall with distance during fictive swimming (triangles of Fig. $4 D$ ). Thus these two methods for estimating the functional length of coupling signals both indicate that coupling signals act over approximately five segments. The EINs have ipsilateral axons that appear to project fewer than five segments (Buchanan et al. 1989). Although these cells may contribute to the coupling observed here, the characteristic loss of silent phases suggests that inhibitory interneurons are of key importance and thus may be due to commissural interneurons with relatively short projections (Ohta et al. 1991). However, it is known that inhibitory interneurons in the lamprey spinal cord can project many segments. For example, some inhibitory CC interneurons have axonal projections up to 30 segments (Buchanan 1982), and inhibitory lateral interneurons have axonal projections up to 50 segments (Rovainen 1974). The results of this study do not imply that these longer-projecting cells have no effects during fictive swimming but suggest that the actions of projections longer than approximately five segments are too weak to rhythmically modulate the ventral root firing. As stated in methods, no relationship was found between the overall length of the preparation and the rate of deterioration of the rhythmic activity with distance. However, there is evidence that longer connections do have effects during fictive swimming. For example, modeling of cross-correlations of ventral root bursting has suggested the presence of long connections (Mellen et al. 1995). In addition, partitioned bath experiments have also shown that longer connections exist (Miller and Sigvardt 1996;Rovainen 1985). In these latter experiments, activity in the middle of a spinal cord piece was blocked while allowing the two ends of the cord to swim fictively. The nonswimming middle region could be extended up to 20 segments with some maintained interactions between the two ends. Although these experiments demonstrate that longer-range connections exist, this study indicates that the local connections are much stronger than the longer-range connections.

\section{Influence of the split cord on the intact cord}

In addition to the presence of neurons in the intact spinal cord region that impose rhythmic activity on the split segments, it was also clear that neurons within the split spinal cord region could influence the activity in the intact cord. These cells would be neurons with ipsilateral axon projections and would likely carry abnormal signals because they originate from segments with deteriorated rhythms (Fig. 8B). When the split regions of the spinal cord were removed from intact cord, not only did the rhythm cease in the split region (caused by the loss of rhythmic input from the intact region), but the quality of rhythmic activity in the intact region improved (caused by removal of disruptive inputs from the split region) (Figs. 5and 7). In most cases (Figs. 4 and 7C), the rhythm in the intact region was more disrupted when the rostral end of the spinal cord piece was split, suggesting that descending ipsilateral neurons have a more powerful influence on the rhythm-generating networks than ipsilateral ascending neurons. Alternatively, the descending projections may be longer than the ascending ones and thus carry signals from the more distant and more disrupted segments of the split region. This would be consistent with the results of partitioned-bath experiments in which the measurement of the distance of projections from an actively swimming one-half of spinal cord into the passive one-half demonstrated that ascending neurons have shorter-range effects during fictive swimming than do descending neurons (Dale 1986).

\section{Comparison with other vertebrates}

In comparison with other vertebrates, the lamprey spinal cord may be an exception with regard to the necessity for commissural interneurons for rhythm generation. In the embryonicXenopus, midline cuts separating the halves of the brain and spinal cord do not abolish rhythmic activity (Kahn and Roberts 1982; Soffe 1989). After separation in theXenopus preparation, rhythmic activity is somewhat faster and more variable than in the intact animal. The persistent rhythmic activity has been postulated to be due to a combination of feedback excitation from motoneurons to produce synchronization and recurrent inhibition from ipsilateral collaterals of commissural interneurons (Roberts et al. 1997). In limbed vertebrates, a direct comparison to lamprey is less clear. In lamprey andXenopus, antagonistic muscle groups are located on opposite sides of the body, whereas in 
limbed vertebrates, antagonistic muscles act on limb joints on the same side of the body. The motoneurons and interneurons associated with these antagonistic groups are also located on the same side of the spinal cord. Thus in limbed vertebrates the cells that would be functionally similar to commissural interneurons of the lamprey, i.e., those providing reciprocal inhibition between antagonist neuronal groups, are not easily accessible for lesion studies as done in this study. There is evidence, however, that commissural interneurons may participate in rhythmic pattern formation in higher vertebrates. For example, in the fictive scratch rhythm of adult turtles (Stein et al. 1995), activity of one hindlimb scratch network generates weak activity in the contralateral limb scratch network, and activation of these commissural projections can in some cases be necessary for the expression of rhythmic activity (Currie and Gonsalves 1997). In the locomotor rhythm of the neonatal rat spinal cord, selective pharmacological activation of one side of the lumbar spinal cord produces rhythmic inhibition in contralateral motoneurons, again demonstrating that commissural interneurons are involved in pattern formation (Kjaerulff and Kiehn 1997). Thus, although commissural interneurons have a role in rhythmic pattern formation in limbed vertebrates, the question of the necessity of reciprocal inhibition between antagonistic neuron groups for rhythm generation remains uncertain.

\section{Conclusions}

The results of this study indicate that commissural interneurons are necessary for rhythm generation of fictive swimming in the lamprey spinal cord. It also suggests that these interneurons can impose rhythmic activity in segments beyond their origin, but this influence is limited to approximately five segments.

I thank D. Komorowski for assistance in some of the experiments.

This work was supported by National Institute of Neurological Disorders and Stroke Grant NS-35725.

\section{FOOTNOTES}

- The costs of publication of this article were defrayed in part by the payment of page charges. The article must therefore be hereby marked "advertisement" in accordance with 18 U.S.C. Section 1734 solely to indicate this fact.

\section{AUTHOR NOTES}

- $\quad$ Address for reprint requests: J. T Buchanan, Dept. of Biology, Marquette University, P. O. Box 1881, Milwaukee, WI 53201-1881.

\section{REFERENCES}

1 Alford S., Williams T. L.Endogenous activation of glycine and NMDA receptors in lamprey spinal cord during fictive locomotion.J. Neurosci.9198927922800

2 Baev K. V., Rusin K. I., Safronov B. V.Primary receptor for inhibitory transmitters in lamprey spinal cord neurons. Neuroscience461992931941

3 Buchanan J. T.Identification of interneurons with contralateral, caudal axons in the lamprey spinal cord: synaptic interactions and morphology.J. Neurophysiol.471982961975

4 Buchanan J. T.Premotor interneurons in the lamprey spinal cord: morphology, synaptic interactions, and activities during fictive swimming. Neurobiology of Vertebrate Locomotion, Grillner S., Stein P.S.G., Stuart D. G., Forssberg H., Herman R. M.1986321334MacMillanLondon

5 Buchanan J. T.Neural network simulation of coupled locomotor oscillators in the lamprey spinal cord.Biol. Cybern.661992367374

6 Buchanan J. T., Grillner S.Newly identified "glutamate interneurons" and their role in locomotion in the lamprey spinal cord.Science2361987312314 
7 Buchanan J. T., Grillner S.A new class of small inhibitory interneurons in the lamprey spinal cord.Brain Res.4381988404407

8 Buchanan J. T., Grillner S., Cullheim S., Risling M.Identification of excitatory interneurons contributing to generation of locomotion in lamprey: structure, pharmacology, and function.J. Neurophysiol.6219895969

9 Buchanan J. T., Kasicki S., Komorowski D.Swim oscillator coupling signals in the lamprey spinal cord.Soc. Neurosci. Abstr.211995687

10 Buchanan J. T., Kasicki S., Quinlan K. A.Effects of motoneuron stimulation in the lamprey spinal cord.Soc. Neurosci. Abstr.2419981666

11 Buchanan J. T., McPherson D. R.The neuronal network for locomotion in the lamprey spinal cord: evidence for the involvement of commissural interneurons.J. Physiol. Paris891995221234

12 Cohen A. H., Ermentrout G. B., Kiemel T., Kopell N., Sigvardt K. A., Williams T. L.Modeling of intersegmental coordination in the lamprey central pattern generator for locomotion.Trends Neurosci.151992434438

13 Cohen A. H., Harris-Warrick R. M.Strychnine eliminates alternating motor output during fictive locomotion in the lamprey.Brain Res.2931984164167

14 Cohen A. H., Wallén P.The neuronal correlate of locomotion in fish. "Fictive swimming" induced in an in vitro preparation of the lamprey spinal cord.Exp. Brain Res.4119801118

15 Currie S. N., Gonsalves G. G.Right-left interactions between rostral scratch networks generate rhythmicity in the preenlargement spinal cord of the turtle.J. Neurophysiol.78199734793483

16 Dale N.Excitatory synaptic drive for swimming mediated by amino acid receptors in the lamprey.J. Neurosci.6198626622675

17 El Manira A., Tegner J., Grillner S.Calcium-dependent potassium channels play a critical role for burst termination in the locomotor network in lamprey.J. Neurophysiol.72199418521861

18 Grillner S., Brodin L., Sigvardt K., Dale N.On the spinal network generating locomotion in lamprey: transmitters, membrane properties and circuitry. Neurobiology of Vertebrate Locomotion, Grillner S., Stein P.S.G., Stuart D. G., Forssberg H., Herman R. M.1986335352MacMillanLondon

19 Grillner S., Wallén P.Does the central pattern generation for locomotion in lamprey depend on glycine inhibition?Acta Physiol. Scand.1101980103105

20 Hagevik A., McClellan A. D.Coupling of spinal locomotor networks in larval lamprey revealed by receptor blockers for inhibitory amino acids: neurophysiology and computer modeling.J. Neurophysiol.72199418101829

21 Homma S., Rovainen C. M.Conductance increases produced by glycine and gamma-aminobutyric acid in lamprey interneurons.J. Physiol. (Lond.)2791978231252

22 Kahn J. A., Roberts A.Experiments on the central pattern generator for swimming in amphibian embryos.Philos. Trans. R. Soc. Lond. B Biol. Sci.2961982229243

23 Kjaerulff O., Kiehn O.Crossed rhythmic synaptic input to motoneurons during selective activation of the contralateral spinal locomotor network.J. Neurosci.17199794339447

24 McPherson D. R., Buchanan J. T., Kasicki S.Effects of strychnine on fictive swimming in the lamprey: evidence for glycinergic inhibition, discrepancies with model predictions, and novel modulatory rhythms.J. Comp. Physiol. [A]1751994311321

25 Mellen N., Kiemel T., Cohen A. H.Correlational analysis of fictive swimming in the lamprey reveals strong functional intersegmental coupling.J. Neurophysiol.73199510201030

26 Miller W. L., Sigvardt K. A.Influence of multisegmental coordinating fibers on rhythmicity of distant segments in the lamprey spinal central pattern generator.Soc. Neurosci. Abstr.2219961642

27 Moore L. E., Hill R. H., Grillner S.Voltage clamp frequency domain analysis of NMDA activated neurons.J. Exp. Biol.17519935987

28 Murphey C. R., Moore L. E., Buchanan J. T.Quantitative analysis of electrotonic structure and membrane properties of NMDA-activated lamprey spinal neurons.Neural Comp.71995486506

29 Ohta Y., Dubuc R., Grillner S.A new population of neurons with crossed axons in the lamprey spinal cord.Brain Res.5641991143148 
30 Oyama Y., Akaike N., Carpenter D. O.Strychnine decreases the voltage-dependent Ca2+ current of both Aplysia and frog ganglion neurons.Cell. Mol. Neurobiol.81988307314

31 Roberts A.How does a nervous system produce behaviour? A case study in neurobiology.Sci. Prog.7419903151

32 Roberts A., Soffe S. R., Perrins R.Spinal networks controlling swimming in hatchling Xenopus tadpoles.Neurons, Networks, and Motor Behavior, Stein P.S.G., Grillner S., Selverston A. I., Stuart D. G.19978389MIT PressBoston, MA

33 Rovainen C. M.Synaptic interactions of identified nerve cells in the spinal cord of the sea lamprey.J. Comp Neurol.1541974189206

34 Rovainen C. M.Effects of groups of propriospinal interneurons on fictive swimming in the isolated spinal cord of the lamprey.J. Neurophysiol.541985959977

35 Shapiro B. I., Wang C. M., Narahashi T.Effects of strychnine on ionic conductances of squid axon membrane.J. Pharmacol. Exp. Ther.18819746676

36 Soffe S. R.Roles of glycinergic inhibition and N-methyl-d-aspartate receptor-mediated excitation in the locomotor rhythmicity of one half of the Xenopus embryo CNS.Eur. J. Neurosci.11989561571

37 Stein P.S.G., Victor J. C., Field E. C., Currie S. N.Bilateral control of hindlimb scratching in the spinal turtle: contralateral spinal circuitry contributes to the normal ipsilateral motor pattern of fictive rostral scratching.J. Neurosci.15199543434355

38 Tabak J., Murphey C. R., Prime L., Tiaho F., Buchanan J. T., Moore L. E.Training models of locomotion network activity.Soc. Neurosci. Abstr.211995143

39 Wallén P., Grillner S.N-Methyl-d-aspartate receptor-induced, inherent oscillatory activity in neurons active during fictive locomotion in the lamprey.J. Neurosci.7198727452755

40 Wallén P., Williams T. L.Fictive locomotion in the lamprey spinal cord in vitro compared with swimming in the intact and spinal animal.J. Physiol. (Lond.)3471984225239

41 Wang X.-J., Rinzel J.Alternating and synchronous rhythms in reciprocally inhibitory model neurons.Neural Comput.419928497

42 Williams T. L.Phase coupling by synaptic spread in chains of coupled neuronal oscillators.Science2581992662665

43 Williams T. L., Sigvardt K. A., Kopell N., Ermentrout G. B., Remler M. P.Forcing of coupled nonlinear oscillators: studies of intersegmental coordination in the lamprey locomotor central pattern generator.J. Neurophysiol.641990862871 